\title{
SURFACE ENHANCED RAMAN SPECTROSCOPY OF HALIDE IONS ON COLLOIDAL SILVER: MORPHOLOGY AND COVERAGE DEPENDENCE
}

\author{
Robin L. GARRELL, Keith D. SHAW and S. KRIMM \\ Department of Physics and Macromolecular Research Center, The University of Michigan, Ann \\ Arbor, Michigan 48109, USA
}

Received 15 June 1982; accepted for publication 15 November 1982

\begin{abstract}
Transmission electron microscopy shows that the colloidal silver used as a substrate for surface enhanced Raman spectroscopy consists of $75-135 \AA$ average diameter faceted crystals, which can be made to aggregate in clusters of a few to thousands of particles. Aggregates are not required for observation of the SER spectrum of adsorbed halide ions or pyridine. Bromide is shown to adsorb more readily than chloride, and in time it displaces chloride on the silver surface. Spectra of halide ions adsorbed on sols with various particle morphologies lend additional support to our interacting dipole model describing the origins of the spectral band fine structure.
\end{abstract}

\section{Introduction}

Surface enhanced Raman spectroscopy (SERS) is an increasingly popular technique for studying molecules and ions adsorbed on metal surfaces. The anomalously intense Raman signals that are obtained (enhancement factors in the range $10^{3}-10^{6}$ ), as well as the applicability to a wide range of adsorbates, make the technique of great interest to analytical and surface scientists. Numerous theories have been proposed to explain the large enhancement (resonance, image field effects, surface plasmons, etc.) [1], each of which presupposes certain morphological characteristics of the surface. In this paper we present an electron microscopic characterization of the colloidal silver system first employed for SERS by Creighton et al. [2] and we explore several factors that influence sol morphology. Our results show that aggregation of sol particles is not required for observation of SER spectra of adsorbed halide ions or pyridine. Enhancement is not increased significantly by the presence of aggregates, contrary to predictions of Moskovits [3], Laor and Schatz [4], and others $[2,5]$.

We recently reported results of halide ion $\left(\mathrm{Cl}^{-}, \mathrm{Br}^{-}\right.$, and $\left.\mathrm{I}^{-}\right)$SERS on silver sols [5]. These and additional experiments using a mixed halide $\left(\mathrm{Cl}^{-}\right.$, $\mathrm{Br}^{-}$) system, incremental halide addition, and sols prepared at elevated 
temperatures are described here in detail, and provide further evidence that the vibrational frequencies and band intensities of adsorbed species are coverage dependent. Though this dependence is in qualitative agreement with the theory of Nichols and Hexter [6], our spectra exhibit a previously unpredicted fine structure that is attributable to the discrete nature of surface coverage structures. We can associate the intensity variations of spectral band components with changes in the relative proportions of the corresponding patterns of dipole interactions. The aggregation-dependent SER spectra of adsorbed $\mathrm{Br}^{-}$ and $\mathrm{Cl}^{-}$are explained by changes in adsorbate coverage fraction and adsorption site preference. A more complete description of the theory and a computer simulation to model the experimentally obtained spectra are presented in the accompanying paper [7].

\section{Experimental}

All solutions were prepared with Burdick and Jackson HPLC grade water. Silver sols were formed, using reagents at 5,22 , or $95^{\circ} \mathrm{C}$, by adding one part $1.0 \mathrm{mM} \mathrm{AgNO}_{3}$ (Fisher ACS grade) to three parts $2.0 \mathrm{mM} \mathrm{LiBH}{ }_{4}$ (Alfa), $\mathrm{NaBH}_{4}$ (Alfa), or $\mathrm{Na}_{2} \mathrm{~S}_{2} \mathrm{O}_{4}$ (Fisher). Addition was dropwise with stirring. Sols could be concentrated to as little as $10 \%$ of their original volume by Amicon filtration or by boiling.

For the halide ion experiments, aliquots of dilute halo-acids (1-50 $\mu 1$ of $0.1-15.0 \mathrm{mM} \mathrm{HCl}, \mathrm{HBr}, \mathrm{HI})$ were added to $0.50 \mathrm{ml}$ portions of sol that had been allowed to age for at least one hour at $20^{\circ} \mathrm{C}$. The mixtures were agitated vigorously for $45 \mathrm{~s}$ prior to placement in $1 \mathrm{~mm}$ diameter borosilicate glass capillaries for the Raman experiments.

Dialyzed samples were prepared using Spectrapore No. 1 dialysis tubing, with three changes of 20 volumes HPLC grade water.

Absorption spectra were recorded on a Cary 118 spectrophotometer.

Specimens were prepared for transmission electron microscopy (TEM) (JEM 100B microscope operated at $80 \mathrm{kV}$ ) by drawing $5 \mu 1$ aliquots of the sol across electrostatically charged carbon-coated grids, followed by rapid drying in air.

Raman spectra were recorded on a spectrometer comprised of a Coherent Radiation Ar ion laser operated at $514.5 \mathrm{~nm}$ and $100 \mathrm{~mW}$; a SPEX 1400 prototype double monochromator with 1800 groove $/ \mathrm{mm}$ holographic gratings, operated at a bandwidth of $1 \mathrm{~cm}^{-1}$; and a cooled ITT FW130 photomultiplier and standard photon counting electronics. Data acquisition, storage, and manipulation (multiple scanning, 17 point smoothing, and background subtraction) utilized a Cromemco Z-2 microcomputer. 


\section{Results and discussion}

\subsection{Sol morphology and surface enhancement}

Transmission electron micrographs of various colloidal silver preparations are shown in fig. 1 . A sol prepared at $5^{\circ} \mathrm{C}$ with $\mathrm{NaBH}_{4}$ as the reducing agent is

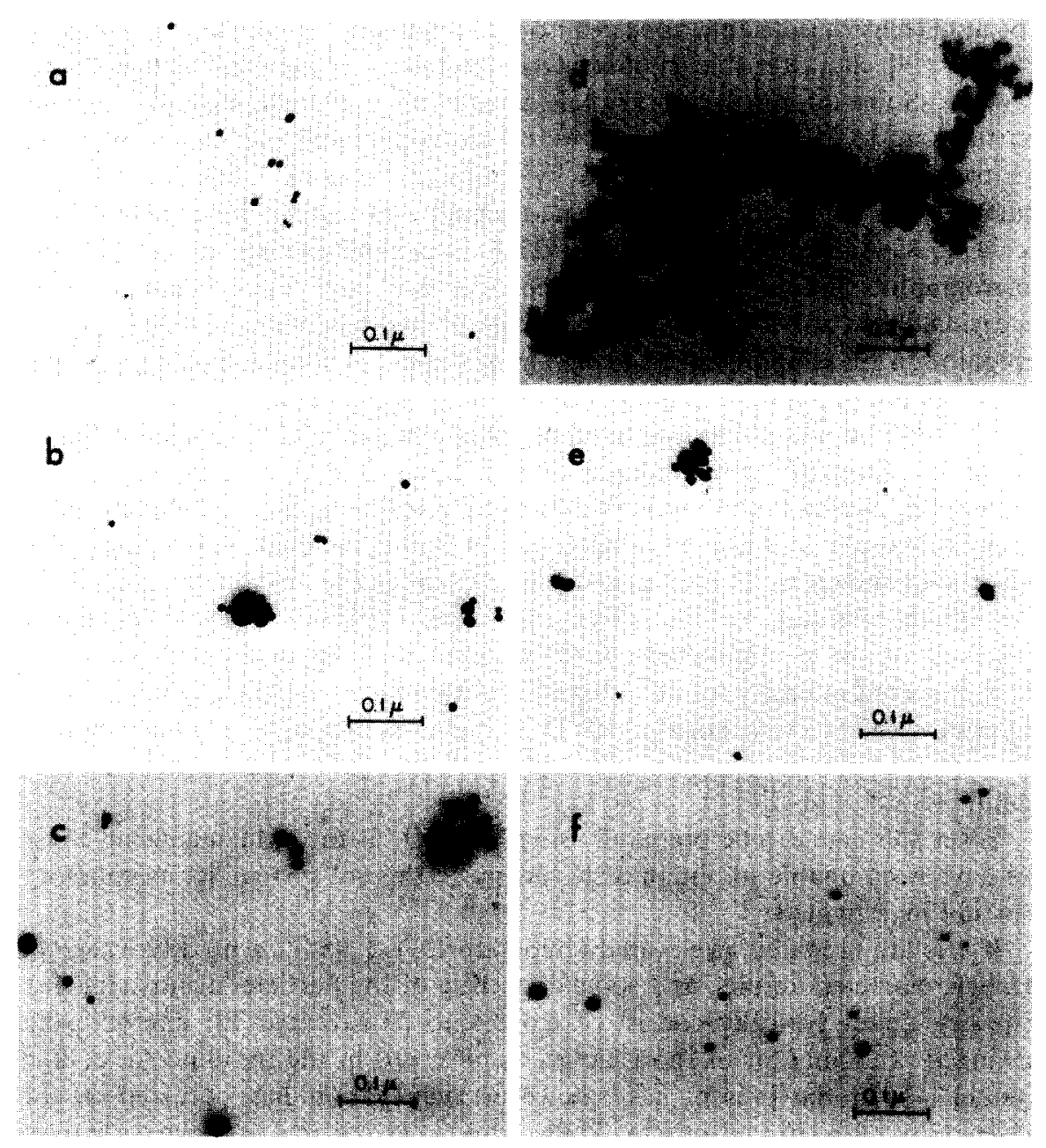

Fig. 1. Transmission electron micrographs of colloidal silver substrates used for SERS: (a) sol prepared at $5^{\circ} \mathrm{C}$ by $\mathrm{NaBH}_{4}$ reduction of $\mathrm{AgNO}_{3}$; (b) sol prepared at $5^{\circ} \mathrm{C}$ by $\mathrm{LiBH}_{4}$ reduction of $\mathrm{AgNO}_{3}$; (c) $\mathrm{LiBH}_{4}$ reduced $\mathrm{AgNO}_{3}$, prepared at $95^{\circ} \mathrm{C}$; (d) particle aggregate resulting from ultrafiltration; (e) sol particles after addition of pyridine; (f) sol particles after addition of $\mathrm{HCl}$. 
comprised of 50-130 $\AA$ (average $75 \AA$ ) diameter particles, such as those seen in fig. 1a. An otherwise identically prepared sol using $\mathrm{LiBH}_{4}$ is shown in fig. $1 \mathrm{~b}$. In the lithium preparation the particles are significantly larger, averaging $135 \AA$ in diameter, but in both cases the particles exhibit sharp edges and well-defined facets of different crystallographic planes. In fact, evidence for the crystalline nature of colloidal silver was obtained by X-ray diffraction as early as 1920 [8], and the presence of different lattice planes on the surface will be seen to be important to the interpretation of the coverage-dependent halide ion spectra. The striations that appear in some sol particles are thickness extinction contours [9], characteristically observed in electron microscopy of wedge-shaped crystals. Some of the larger crystals appear as truncated triangles. Similar particles up to $2500 \AA$ in diameter were observed in gold sols by Milligan and Morriss [10], and were designated by Suito as "trigons" [11]. Most of the particles are cuboctahedral or nugget-shaped, and exhibit roughness on the scale of $25 \AA$. Roughness less than $25 \AA$ is beyond the resolution of these micrographs. Since in these specimens the particles occur singly or very occasionally in small clusters (an example of which is shown in fig. 1b) the preparation of samples for TEM apparently did not induce aggregation, nor did it disrupt pre-existent aggregates, as will be seen below.

It should be noted that, for the most part, the particles have axial ratios of 1.0 to 1.5 . Electrodynamic theories of SERS in colloidal systems [12] rely on large eccentricities (axial ratios $>2.0$ ) to explain the significant enhancement that is observed at relatively long (e.g. $514.5 \mathrm{~nm}$ ) incident wavelengths. For adsorbed halide ions it is not possible to determine the absolute magnitude of the enhancement, since the silver surface is intrinsically involved in the observed vibration. Assessment of the incident wavelength dependence of the SER scattered intensity for adsorbates would however test the validity of these theories. Other factors, such as $10-15 \AA$ scale roughness, or consideration of the surface aspect ratios of the spheriods, may account for additional enhancement.

Silver sols can also be prepared using $\mathrm{Na}_{2} \mathrm{~S}_{2} \mathrm{O}_{3}$ as the reducing agent. Since we have been unable to obtain SER spectra in this sol system, its morphology was not investigated.

If sols are prepared at elevated temperatures, e.g. $95^{\circ} \mathrm{C}$, a broader range of particle diameters results, and some clustering of the particles occurs (fig. 1c). The aggregates typically consist of from 4 to 20 (average 10) microcrystals arranged randomly, or in short chains as observed in the pyridine-Au colloid system [4]. In the present case, however, aggregation has occurred in the absence of a specific adsorbate. Thus, adsorbates are not required to induce clustering of the sol particles.

Concentration by membrane ultrafiltration (Amicon UM05) to 20\% of the original hydrosol volume also results in aggregation. Many single particles persist in such concentrates along with aggregates such as those in fig. 1c and a 
few very large aggregates such as is shown in fig. 1d. By comparison, dialysis and concentration by boiling result in sols comprised almost exclusively of this type of aggregate containing tens to hundreds of microcystals. The highly aggregated sols exhibit visible absorption spectra in which the $390 \mathrm{~nm}$ peak attributable to small particle resonant Mie scattering [2] is substantially diminished in intensity, while the plasmon absorption band broadens and shifts to longer wavelengths $(700 \mathrm{~nm})$ with increasing particle size. We found these sols to be rather unstable, precipitation occurring within several hours after preparation or immediately following the addition of adsorbates such as pyridine.

Early experimental studies on silver sols had indicated that aggregation of the collodial particles was required for observation of SERS of adsorbed species such as pyridine [2-4]. Von Raben et al. [13] recently reported SERS of cyanide complexes adsorbed on predominantly unaggregated colloidal gold spheres which suggests that the SER phenonmenon in colloidal systems does not depend on the presence of aggregates. Of course, there is the possibility that the entire observed Raman signal was due to scattering from the few aggregates which were present.

To assess the nature and extent of adsorbate-induced aggregation, we investigated the morphology of silver sols containing adsorbed halide ions or pyridine. A typical micrograph of a SER-scattering sol with pyridine is shown in fig. le. As with von Raben's gold sols, the larger portion of the particles are unaggregated. In this case, there was no apparent color change upon addition of the adsorbate, and similar preparations for which visible absorption spectra have been measured show no significant spectral differences before and after adsorbate addition. (Addition of larger amounts of pyridine induces the color changes described by Creighton et al. with concomitant shifting and broadening of the visible absorption bands.) The few aggregates that are present are small, consisting of 10 to 40 particles. We did not, however, evaluate the exact percentage of sol particles present in the aggregates.

Micrographs of sols containing $\mathrm{HX}$, such as fig. 1f, show predominantly single particles, with very occasional clusters of up to six spheroids. Unlike the pyridine-adsorbed particles, these appear somewhat rounded and "coated". This coating probably consists of halide salts that have dried onto the silver particles. Nevertheless, the scarcity of aggregates suggests that clustering of the colloid particles is not required for the observation of SER spectra of halide ions.

Clearly the addition of adsorbates does not necessarily result in extensive aggregation of the colloidal silver particles. The question of whether aggregation is required for the observation of SER scattering (e.g. by pyridine) in colloidal systems was addressed by determining the contribution to the total Raman scattering from molecules adsorbed on aggregates in a partially aggregated system. This was done by removing aggregates from the adsorbate-con- 
taining sol by microfiltration (using Nuclepore polycarbonate, $0.05 \mu \mathrm{m}$ pore size; smaller pore size filters clog rapidly). We examined the filtrates by TEM, and confirmed the nearly complete removal of aggregates. SER spectra of the ring modes of adsorbed pyridine before and after filtration showed only a $20 \%$ decrease in scattered intensity. The color intensity of the sols diminished following filtration, so we attribute the decreased Raman signal to a reduced number of scattering particles in the beam, a consequence of retention of the aggregated and some unaggregated colloidal particles in the microfilter. We therefore believe that molecules adsorbed on unaggregated particles are responsible for most of the SER signal.

\subsection{Spectroscopy}

Both theoretical and experimental studies have suggested that the extent, density, and homogeneity of surface coverage influence the observed SER frequencies and scattered intensities [6,7,14-16]. Using electrochemical cells, Wetzel, Gerischer and Pettinger [17] observed voltage-dependent silver-halide vibrations at $246 \mathrm{~cm}^{-1}(\mathrm{Ag}-\mathrm{Cl}), 155 \mathrm{~cm}^{-1}(\mathrm{Ag}-\mathrm{Br})$ and $117 \mathrm{~cm}^{-1}(\mathrm{Ag}-\mathrm{I})$. Frequency shifts were attributed to a weakening of the $\mathrm{Ag}-\mathrm{X}$ bond with more negative applied potential. Nichols and Hexter [6] have predicted that the $\mathrm{Ag}-\mathrm{Cl}$ stretching vibration frequency should vary continuously as a function of surface coverage. The fine structure in our partial coverage SER spectra [5] of halide ions adsorbed on colloidal silver surfaces showed, however, that the surface coverage structures are discrete in nature.

Studies of the time-dependence of mixed-halide-plus-sol spectra, the concentration-dependence of halide ion adsorption on colloidal silver, and halides adsorbed on sols prepared at elevated temperatures now lend additional support to our model of surface coverage. In this model the intensity variations of spectral band components are associated with changes in the relative proportions of the specific surface dipole arrangements that give rise to particular frequencies. Both the observed shifts in the mean band frequencies with changes in coverage as well as the fine structure of the bands can be explained by this interacting-dipole model, which is detailed in the accompanying paper.

Before discussing the spectral effects of partial halide ion coverage, we note that the cation seems to have essentially no effect on the spectrum. We have made high coverage $\mathrm{Cl}^{-}$sols in the presence of $\mathrm{H}^{+}, \mathrm{Li}^{+}, \mathrm{Na}^{+}, \mathrm{K}^{+}, \mathrm{Tl}^{+}$, $\mathrm{N}\left(\mathrm{CH}_{3}\right)_{4}^{+}, \mathrm{Ca}^{2+}, \mathrm{Ba}^{2+}$, and $\mathrm{Y}^{3+}$ ions, and we find no significant change in the $\mathrm{Cl}^{-}$frequency (the mean position of the band maximum for the above ions is $242.2 \pm 1.6 \mathrm{~cm}^{-1}$ ). This suggests that the cations do not influence the $\mathrm{Ag}-\mathrm{Cl}^{-}$vibration.

Partial coverage spectra of mixed $\mathrm{Br}^{-}-\mathrm{Cl}^{-}$systems have been discussed previously [5]. The actual replacement of $\mathrm{Cl}^{-}$by $\mathrm{Br}^{-}$on the silver surface is 
demonstrated by the sequence of spectra in fig. 2. The first spectrum obtained from a series of scans of a sol to which excess (for monolayer coverage) $\mathrm{Cl}^{-}$ and $\mathrm{Br}^{-}$had been added simultaneously is given by fig. 2a, corresponding to $t=0 \mathrm{~h}$ from sample preparation; subsequent scans at $t=7,23$, and $144 \mathrm{~h}$ are given in figs. $2 \mathrm{~b}, 2 \mathrm{c}$, and $2 \mathrm{~d}$, respectively. "Full" coverage $\mathrm{Br}^{-}$and $\mathrm{Cl}^{-}$spectra for sols prepared at $20^{\circ} \mathrm{C}$ are given in figs. $3 \mathrm{a}$ and $3 \mathrm{e}$ respectively. Fig. 3 also shows spectra of $\mathrm{Br}^{-}$and $\mathrm{Cl}^{-}$respectively adsorbed on: (1) sol prepared at $95^{\circ} \mathrm{C}$ and concentrated by heating (b and f); (2) sol prepared at $20^{\circ} \mathrm{C}$ and concentrated by heating ( $\mathrm{c}$ and $\mathrm{g}$ ); (3) sol prepared at $20^{\circ} \mathrm{C}$, with less than monolayer quantity of $\mathrm{Br}^{-}$and excess $\mathrm{Cl}^{-}$( $\mathrm{d}$ and $\mathrm{h}$ ).

As the fig. $2 \mathrm{Cl}^{-}$band centered near $236 \mathrm{~cm}^{-1}$ (which is below the 248 $\mathrm{cm}^{-1}$ of the "full" coverage $\mathrm{Cl}^{-}$spectrum) decreases in intensity with time, the bromide band centered near $168 \mathrm{~cm}^{-1}$ increases in intensity, demonstrating replacement of $\mathrm{Cl}^{-}$by $\mathrm{Br}^{-}$on the surface. This obviously indicates a favorable free energy change upon adsorption of $\mathrm{Br}^{-}$and desorption of $\mathrm{Cl}^{-}$, though the details of the process are not known. In particular, there is a

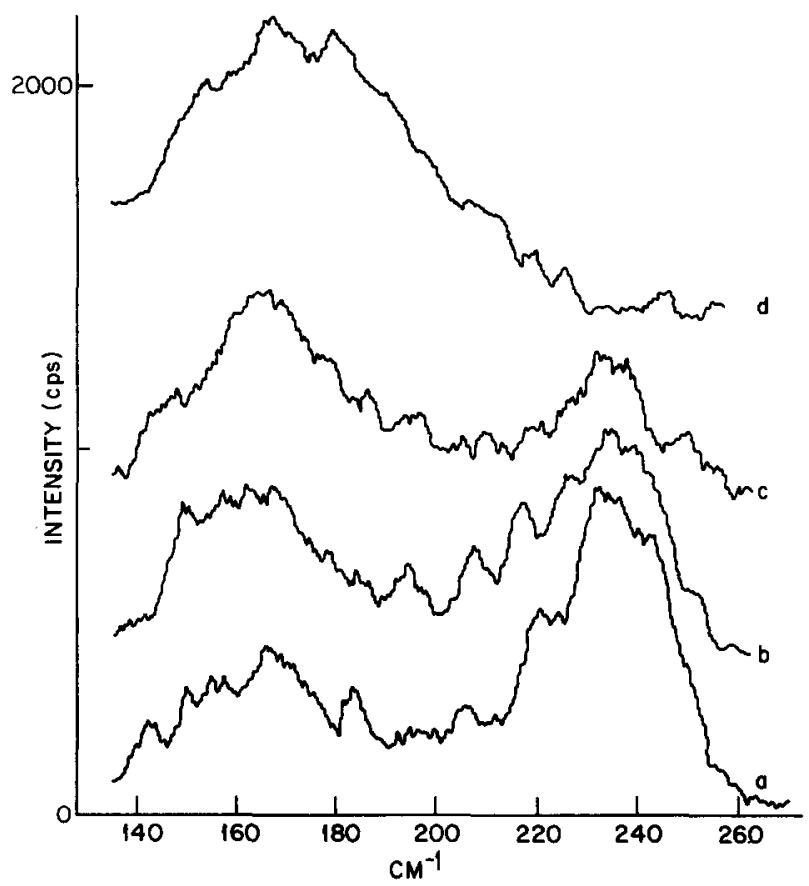

Fig. 2. Time-dependent replacement of $\mathrm{Cl}^{-}$by $\mathrm{Br}^{-}$on the colloidal silver surface. Large excess $\mathrm{Cl}^{-}$for monolayer coverage, plus sub-monolayer quantity of $\mathrm{Br}^{-}$added to sol prepared by $\mathrm{LiBH}_{4}$ reduction of $\mathrm{AgNO}_{3}$ at $5^{\circ} \mathrm{C}$ : (a) immediately after $\mathrm{HX}$ addition; (b) $t=7 \mathrm{~h}$; (c) $t=23 \mathrm{~h}$; (d) $t=144 \mathrm{~h}$. Each spectrum is the sum of four scans ( $2 \mathrm{~s}$ photon accumulation per $0.5 \mathrm{~cm}^{-1} \mathrm{step}$ ), to which one 17-point smoothing function has been applied. 


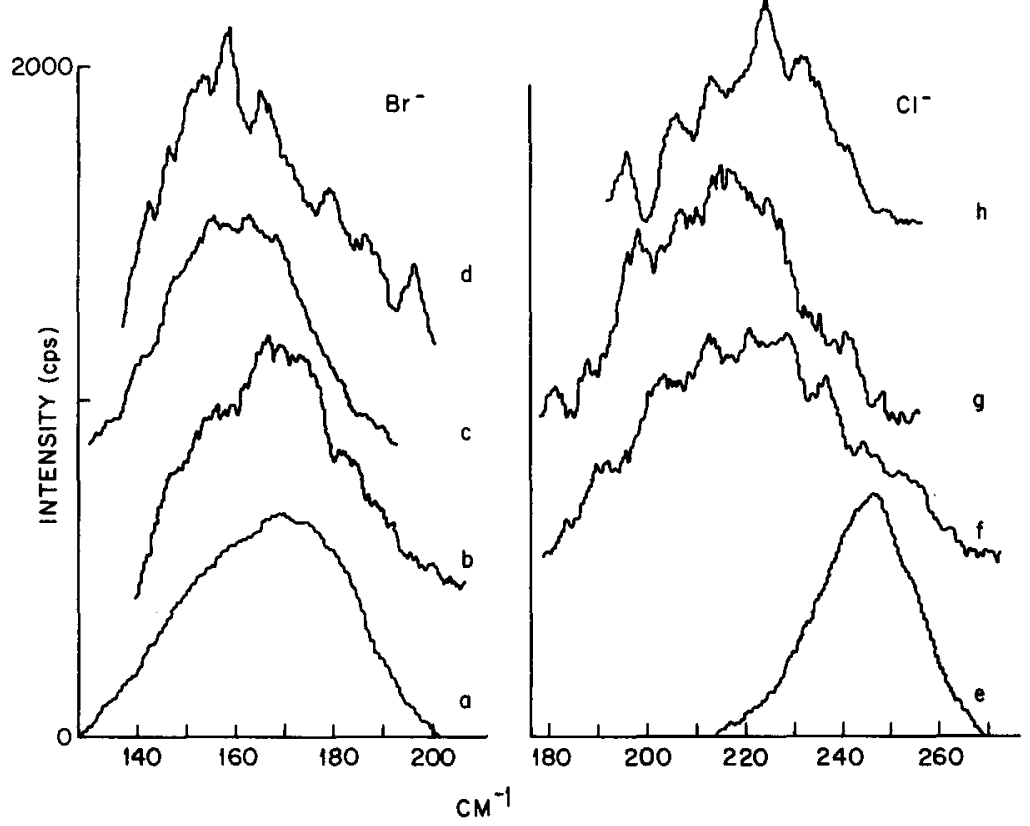

Fig. 3. Halide ion spectra for various sol preparations: (a) $\mathrm{Br}^{-}$on sol prepared at $20^{\circ} \mathrm{C}$ (high coverage); (b) $\mathrm{Br}^{-}$and $\mathrm{Cl}^{-}$together added to sol prepared at $100^{\circ} \mathrm{C}$; (c) $20^{\circ} \mathrm{C}$ prepared sol, concentrated by boiling to $7 \%$ of original volume, to which $\mathrm{Br}^{-}$was then added; (d) less than monolayer quantity $\mathrm{Br}^{-}$and excess $\mathrm{Cl}^{-}$together added to sol prepared at $20^{\circ} \mathrm{C}$ (partial coverage $\mathrm{Br}^{-}$); (e) $\mathrm{Cl}^{-}$on sol prepared at $20^{\circ} \mathrm{C}$ (high coverage); (f) $100^{\circ} \mathrm{C}$ prepared sol, concentrated by boiling to $15 \%$ of original volume, to which $\mathrm{Cl}^{-}$was then added; (g) $5^{\circ} \mathrm{C}$ prepared sol concentrated by boiling to $4 \%$ of original volume, to which $\mathrm{Cl}^{-}$was then added; (h) see (d) (partial coverage $\mathrm{Cl}^{-}$). Each spectrum is the sum of four scans (1-2 s photon accumulation per $0.5 \mathrm{~cm}^{-1} \mathrm{step}$ ), to which one 17-point smoothing function has been applied. Spectrum (e) has been drawn at one-half its true vertical scale.

significant increase in intensity on the high frequency side of the $\mathrm{Br}^{-}$band with retention of fine structure, evidence for an increased incidence of higher coverage $\mathrm{Br}^{-}$surface structures [5,7]. The band near $196 \mathrm{~cm}^{-1}$ could arise from either high coverage $\mathrm{Br}^{-}$structures or low coverage $\mathrm{Cl}^{-}$structures. Similarly, comparing fig. $2 \mathrm{a}$ to fig. $3 \mathrm{e}$ there is a definite absence of high coverage $\mathrm{Cl}^{-}$structures, as evidenced by the lower intensity above $240 \mathrm{~cm}^{-1}$ in fig. 2a. The fairly uniform diminution of the $\mathrm{Cl}^{-}$band from fig. $2 \mathrm{a}$ to fig. $2 \mathrm{~d}$ (again with no loss of fine structure) indicates a decreased incidence of both high and low coverage structures. By $23 \mathrm{~h}$, fig. $2 \mathrm{c}$, very few high coverage $\mathrm{Cl}^{-}$ structures are present at all, and by $144 \mathrm{~h}$, fig. $2 \mathrm{~d}$, ostensibly all $\mathrm{Cl}^{-}$ions have desorbed.

Thus, the notion that higher frequency portions of the halide bands are 
attributable to local higher coverage halide structures on the silver surfaces, with lower frequency portions being associated with lower coverage structures, is supported by the spectral changes that are observed in the course of time in mixed halide experiments. This does not, however, give information as to the manner in which the ions are initially adsorbed: gradually and completely randomly over the entire available surface, in small clusters (islands) which grow larger with further addition of ions, or suddenly over the entire surface to the maximum coverage allowable on a silver lattice.

We therefore examined sols to which $\mathrm{Br}^{-}$or $\mathrm{Cl}^{-}$was added incrementally, starting with a quantity of halo-acid well below that required for monolayer coverage. The results are shown in fig. 4 , displayed as intensity of the SERS band (maximum peak height, irrespective of exact position) versus total added halo-acid. These results are quite reproducible for a given sol (note error bars) and the curve shfits only slightly when different sol preparations are used.

The extent of aggregation was monitored for each sample by visible absorbance spectroscopy concomittant with SERS. Samples which gave up to and including the maximum SER intensity showed absolutely no change in the visible absorption spectrum from $300-800 \mathrm{~nm}$, indicating that the amount of induced aggregation was negligible. This is in agreement with results obtained for adsorbed pyridine (vide supra). In fact, addition of $\mathrm{HCl}$ to sols causes a significant narrowing and slight blue-shifting of the absorption band (from 65 nm FWHM to $40 \mathrm{~nm}$ FWHM, peak maximum shifted $2 \mathrm{~nm}$ from $396 \mathrm{~nm}$ ),

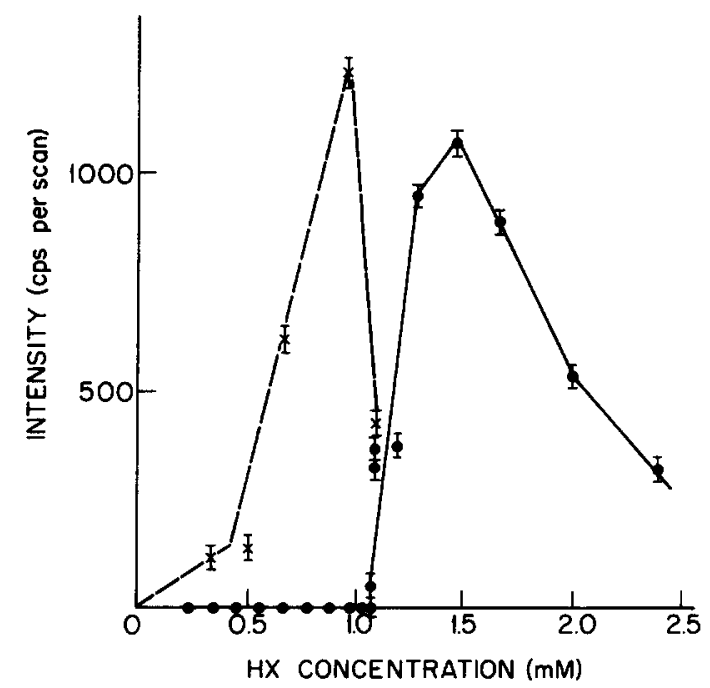

Fig. 4. Raman intensity of $\mathrm{Cl}^{-}\left(240 \mathrm{~cm}^{-1}\right)(\Theta)$ and $\mathrm{Br}^{-}\left(167 \mathrm{~cm}^{-1}\right)(\times)$ bands as a function of added halo-acid. 
whereas addition of pryridine causes no broadening and a $2 \mathrm{~nm}$ red-shifting. Apparently $\mathrm{Cl}^{-}$adsorption drastically alters the optical properties of the individual sol particles, but we cannot describe these changes in detail. If $\mathrm{Cl}^{-}$ is added to a sol which is known to be aggregated (for example, induced by heat concentration, and assessed by visible absorbance spectroscopy), the resultant SER band is slightly less intense than that obtained for an unaggregated sol, and is similar in bandshape to low-coverage $\mathrm{Cl}^{-}$spectra [5].

Halides were also added to sols from which aggregates had been removed by microfiltration. TEM of samples which gave up to the maximum SER intensity showed no evidence of particle aggregation.

The adsorption process clearly cannot be described either as gradual and random, or as island formation, as these would result in a steady increase in the SERS intensity with halide addition. Wetzel et al. [17] observed just such a gradual adsorption of halide ions on silver electrodes as a function of applied voltage. In our case, a minimum solution concentration is required for significant adsorption to occur, and this concentration represents a quantity more than ten times the amount estimated to be required for monolayer coverage on the available silver surface. Bromide adsorbs at a lower threshold concentration than $\mathrm{Cl}^{-}$, consistent with the time-dependent replacement of $\mathrm{Cl}^{-}$by $\mathrm{Br}^{-}$ on the surface (see fig. 2). This indicates that $\mathrm{Br}^{-}$has a more negative enthalpy of adsorption on silver, not unreasonable in light of the lower solubility of $\mathrm{AgBr}$ compared with $\mathrm{AgCl}$. Both sets of spectra exhibit a sharp decrease in intensity at high adsorbate concentrations, due to the reduced number of scattering particles in the beam that results from aggregation and precipitation of the sol particles. Visible absorption spectra of such samples exhibit the decreased intensity and broadening of the $396 \mathrm{~nm}$ band, and the increased intensity from 420 to $800 \mathrm{~nm}$, characteristic of aggregation [2].

The ordering of adatoms on surfaces due to repulsive interactions is common. In some cases, such as $\mathrm{CO}$ on $\mathrm{Ni}(100)$ at $>50 \%$ coverage [18], the repulsive adsorbate/adsorbate interaction can dominate the adsorbate/solid interaction to the point where the adsorbate develops a structure out of register with the substrate, i.e. an incommensurate lattice. While it is not clear whether halide ion superlattices are incommensurate with those of the silver substrate, experiments on sols manipulated at elevated temperatures suggest that several types of adsorbate superlattices are formed.

In fig. 3 the partial coverage $\mathrm{Br}^{-}$spectrum (d), compared with the "full" coverage spectrum (a), shows the predicted coverage-dependent increase in intensity in the low frequency region and decrease in the high frequency region $[5,7]$. The band as a whole (roughly $130-200 \mathrm{~cm}^{-1}$ ) does not shift downward in frequency. The partial coverage $\mathrm{Cl}^{-}$spectrum (h) also shows greater intensity in the low frequency portion of the band, compared with the full coverage spectrum (e). (Recall that the intensity near $196 \mathrm{~cm}^{-1}$ might be attributable to $\mathrm{Br}^{-}$or to $\mathrm{Cl}^{-}$structures.) The spectra for $\mathrm{Cl}^{-}$on sols 
concentrated by heating ( $\mathrm{f}$ and $\mathrm{g}$ ), hence aggregated, show a dramatic increase in intensity between 180 and $210 \mathrm{~cm}^{-1}$, well below the frequencies observed for partial $\mathrm{Cl}^{-}$coverage on the unaggregated sol. This change is not observed for $\mathrm{Br}^{-}$, which suggests that a change in the particle facets has occurred which has in turn affected $\mathrm{Cl}^{-}$adsortption but not $\mathrm{Br}^{-}$adsorption. Note also that spectra $b$ and $f$ from sols prepared at $95^{\circ} \mathrm{C}$ exhibit considerably higher intensity in the high frequency regions (above $180 \mathrm{~cm}^{-1}$ for $\mathrm{Br}^{-}$, and above $245 \mathrm{~cm}^{-1}$ for $\mathrm{Cl}^{-}$) compared with $\mathrm{c}$ and $\mathrm{g}$, as well as with $\mathrm{d}$ and $\mathrm{h}$. These spectra emphasize the fact that the increase in low-frequency intensity in the concentrated sols with $\mathrm{Cl}^{-}$is not attributable simply to a decrease in $\mathrm{Cl}^{-}$ coverage.

Aggregation clearly causes changes in the silver sol surface which result in substantial changes in halide adsorption. Theoretical modeling of silver (100) and (111) surfaces [7] corroborates the idea that changes in the availability of certain types of adsorption sites result from particle aggregation. Decreasing average facet size, or decreasing coverage fraction, causes the bands to be shifted downward in frequency and broadened. The observed changes in $\mathrm{Br}^{-}$ spectra $b$ and $c$ can be attributed to a decrease in coverage fraction. The breadth of the $\mathrm{Cl}^{-}$band in $f$ and $g$ could be a consequence of contributions from $\mathrm{Cl}^{-}$surface structures on a wide range of facet sizes. The intensity in the region $180-210 \mathrm{~cm}^{-1}$ in these two spectra may not be attributable solely to adsorption on smaller particle facets, however, since a comparable effect would be expected for the $\mathrm{Br}^{-}$band in spectra $\mathrm{b}$ and $\mathrm{c}$. This is not observed.

The interpretation of the spectra in fig. 3 is complicated by the number of variables involved: particle size distribution, the nature and extent of aggregation, and the difficulty of assessing the exact halide ion surface coverage fraction, to name but a few. Nevertheless these studies suggest possibilities for using SERS not only to investigate particular adsorbates, but also to characterize both the substrate metal surface and the adsorption process itself at the aqueous-metal interface.

\section{Conclusions}

Using TEM, we have investigated the morphology of the colloidal silver particles used as a substrate for SERS. Aggregation of these 50 to $200 \AA$ spheroidal (axial ratios 1.0-1.5) particles is not necessarily a consequence of the addition of adsorbates such as halide ions or pyridine, nor is it required for the observation of SER spectra. Bromide is shown to adsorb more readily than $\mathrm{Cl}^{-}$at the surface, and in time displaces adsorbed $\mathrm{Cl}^{-}$. Spectra of halides adsorbed on aggregated sols give information both on the extent of halide ion coverage of the surface, and on the site preferences of $\mathrm{Cl}^{-}$and $\mathrm{Br}^{-}$adsorbed on silver at the aqueous interface. 


\section{Acknowledgements}

We thank G.W. Ford, J.P. Langmore, J.W. Schwank, and W.H. Weber for helpful discussions. This work was supported by National Science Foundation grants DMR 7800753 and PCM 79-21652, and by the Macromolecular Research Center (R.L.G.).

\section{References}

[1] See, for example, recent reviews and references therein:

(a) J.A. Creighton, in: Springer Series in Chemical Physics, Vol. 15, Ed. R.F. Willis (Springer, New York, 1980) p. 145;

(b) R.P. Van Duyne, in: Chemical and Biochemical Applications of Lasers, Vol. 4, Ed. C.B. Moore (Academic Press, New York, 1979) p. 101.

[2] J.A. Creighton, C.G. Blatchford and M.G. Albrecht, J. Chem. Soc. Faraday II, 75 (1979) 790.

[3] M. Moskovits, J. Chem. Phys. 69 (1978) 4159.

[4] U. Laor and G.C. Schatz, Chem. Phys. Letters 82 (1981) 566.

[5] R.L. Garrell, K.D. Shaw and S. Krimm, J. Chem. Phys. 75 (1981) 4155.

[6] H. Nichols and R.M. Hexter, J. Chem. Phys. 74 (1981) 2059.

[7] K.D. Shaw, R.L. Garrell and S. Krimm, Surface Sci. 124 (1983) 625.

[8] H.B. Weiser, Inorganic Colloid Chemistry, Vol. 1 (Wiley, New York, 1933) p. 131.

[9] G. Thomas, Transmission Electron Microscopy of Metals (Wiley, New York, 1962).

[10] W.O. Milligan and R.H. Morriss, J. Am. Chem. Soc. 86 (1964) 3461.

[11] E. Suito and N. Ueda, J. Electron Microsc. (Tokyo) I (1953) 33.

[12] M. Kerker, Pure Appl. Chem. 53 (1981) 2083.

[13] K.U. von Raben, R.K. Chang and B.L. Laube, Chem. Phys. Letters 79 (1981) 465.

[14] G.D. Mahan and A.A. Lucas, J. Chem. Phys. 68 (1978) 1344.

[15] G.L. Eesley and J.R. Smith, Solid State Commun. 31 (1979) 815.

[16] G.L. Eesley, Phys. Letters A 81 (1981) 193.

[17] H. Wetzel, H. Gerischer and B. Pettinger, Chem. Phys. Letters 78 (1981) 392.

[18] S.R. Morrison, The Chemical Physics of Surfaces (Plenum, New York, 1977) p. 236. 